\title{
Estudo do Comportamento Térmico e Mecânico do PLA Modificado com Aditivo Nucleante e Modificador de Impacto
}

\author{
Renato Brisigueli Pereira, Ana Rita Morales \\ Departamento de Engenharia de Materiais e de Bioprocessos - DEMBio, \\ Faculdade de Engenharia Química, UNICAMP
}

\begin{abstract}
Resumo: O poliácido lático (PLA) é, dentre os polímeros biodegradáveis, o que apresenta o maior potencial para substituição aos polímeros derivados do petróleo na indústria de embalagens. No entanto uma de suas principais limitações está na baixa estabilidade térmica dimensional. O presente trabalho apresenta um estudo da influência da utilização de dois aditivos: modificador térmico (nucleante) e modificador de impacto, na Temperatura de Distorção Térmica (HDT), no Ponto de Amolecimento Vicat (Vicat) e nas propriedades mecânicas do PLA. Os mecanismos de atuação destes aditivos foram estudados por alterações na temperatura de transição vítrea (Tg) e na cristalinidade medidas por calorimetria diferencial exploratória (DSC) em corpos de prova injetados sem e com tratamento de recozimento. Aplicou-se um planejamento experimental para tratamento estatístico dos dados. Os valores de HDT e de Vicat foram pouco alterados pela presença dos aditivos, embora tenha sido observado aumento nos valores do módulo elástico de tração. O tratamento de recozimento foi responsável pelo aumento tanto na HDT como na Vicat devido ao aumento no grau de cristalinidade. As curvas de DSC mostraram uma grande complexidade no comportamento térmico do PLA, sendo fortemente influenciado pelas condições de tratamento térmico.
\end{abstract}

Palavras-chave: PLA, aditivos nucleantes, propriedades mecânicas, propriedades térmicas.

\section{Study of Mechanical and Thermal Behavior of PLA Modified with Nucleating Additive and Impact Modifier}

\begin{abstract}
Poly(lactic acid) (PLA) is the biodegradable polymer with greatest potential to replace petroleum-based polymers for the packaging industry. However one of its major limitations is the poor thermal dimensional stability. This paper presents a study of the influence of two additives: thermal modifier (nucleating) and impact modifier on the Heat Distortion Temperature (HDT), Vicat Softening Temperature (Vicat) and mechanical properties of PLA. The mechanism of these additives was evaluated by analyzing the glass transition temperature (Tg) and degree of crystallinity by Differential Scanning Calorimetry (DSC) in the samples with and without annealing treatment. An experimental design was applied for the statistical treatment of the data. The values of HDT and Vicat were slightly altered by the presence of additives, although the tensile elastic modulus increased. Annealing was responsible for the increase in HDT as well as in Vicat due to an increased degree of crystallinity. DSC curves showed a great complexity in the thermal behavior of PLA, being strongly influenced by the conditions of heat treatment.
\end{abstract}

Keywords: PLA, nucleating additives, mechanical properties, thermal properties.

\section{Introdução}

O poliácido lático (PLA) apresenta-se como um grande promissor na substituição dos polímeros derivados do petróleo. Sua aceitação tanto no meio científico como mercadológico é um fator encorajador para o desenvolvimento de novos produtos biodegradáveis, tornando-se competitivo para aplicações em embalagens em geral $^{[1]}$.

A produção do PLA apresenta inúmeras vantagens: pode ser obtido de fontes agrícolas renováveis, sua produção consome quantidades consideráveis de dióxido de carbono, é reciclável e compostável, sendo uma alternativa do ponto de vista ambiental e apresenta potencial para melhorar a economia nas propriedades agrícolas $^{[1]}$. Porém este material puro apresenta algumas limitações importantes de aplicação e por isto são encontrados na literatura vários estudos para melhorar suas propriedades físicas e mecânicas tais como flexibilidade, resistência ao impacto e processamento através de aditivação ${ }^{[2-5]}$, da preparação de blendas com outros polímeros $^{\left[{ }^{[6]}\right.}$ ou de reticulação ${ }^{[7]}$.

Embalagens termoformadas são produzidas em grande quantidade e a possibilidade em produzi-las com um polímero biodegradável seria de grande relevância do ponto de vista ambiental. Porém uma limitação para esta aplicação está na falta de estabilidade térmica do PLA, e embalagens feitas com este material podem apresentar deformações mesmo quando submetidas às temperaturas de transporte. Considerando que a distorção sob o efeito da temperatura estaria ocorrendo em temperaturas próximas à Tg do PLA, que é descrita ocorrer tipicamente

Autor para correspondência: Ana Rita Morales, Departamento de Engenharia de Materiais e de Bioprocessos, Faculdade de Engenharia Química, Universidade Estadual de Campinas - UNICAMP, Av. Albert Einstein, 500, CEP 13083-852, Campinas, SP, Brasil, e-mail:morales@feq.unicamp.br 
de $50{ }^{\circ} \mathrm{C} \mathrm{a} 80{ }^{\circ} \mathrm{C}^{[1]}$ e que o grau de cristalinidade também poderia ser um fator predominante para este efeito, a modificação com aditivo nucleante foi estudada neste trabalho. Um modificador de impacto também foi considerado para flexibilizar as cadeias reduzindo o nível de tensionamento nas embalagens e com isto diminuir o efeito de distorção pelo aquecimento das embalagens em uso, além de facilitar o processo de corte necessário no processo das embalagens termoformadas.

O objetivo deste trabalho foi o de estudar as alterações na Temperatura de Deflexão Térmica (HDT) e na Temperatura de Amolecimento Vicat (Vicat) do PLA causadas pelas presenças dos aditivos nucleante e modificador de impacto. Paralelamente também foram estudadas as propriedades mecânicas. O estudo dos mecanismos de ação dos aditivos foi feito analisando-se as transições térmicas, Temperatura de transição vítrea (Tg), Temperatura de cristalização (Tc) e Temperatura de fusão (Tm) nos sistemas, pois o comportamento térmico do PLA é descrito como altamente sensível à história térmica e às condições de processamento ${ }^{[8]}$.

\section{Materiais e Métodos}

O poliácido lático (PLA) usado neste trabalho foi o Ingeo 2002D da Cargill Dow, fabricado pela Natureworks, que apresenta densidade de $1,24 \mathrm{~g} / \mathrm{cm}^{3}$, índice de fluidez de 5 a $7 \mathrm{~g} / 10 \mathrm{~min}$ a $210{ }^{\circ} \mathrm{C} / 2,16 \mathrm{~kg}$, teor de $\mathrm{L}$ de 95-96\% e massa molar de $205.000 \mathrm{~g} / \mathrm{mol}$. Os aditivos utilizados foram o modificador térmico (MT) com aditivo nucleante, Biomax Thermal 300 da DuPont, cujos componentes descritos pelo fornecedor são: copolímero de etileno/acrilato $(>40 \%)$, n-butil/acrilato $(<0,1 \%)$ e cera inerte $(<60 \%)$, com densidade de $0,94 \mathrm{~g} / \mathrm{cm}^{3}$ e ponto de fusão de $113{ }^{\circ} \mathrm{C}$; e o modificador de impacto (MI) Biostrenght 280 da Arkema que é um composto de ácidos e ésteres orgânicos e foi fornecido como um concentrado incorporado a 1:1 (m/m) em PLA, desenvolvido para aumentar a resistência ao impacto do PLA.

A incorporação dos aditivos na matriz polimérica de PLA foi feita diretamente no processo de injeção dos corpos de prova. O PLA foi submetido à secagem prévia a $100{ }^{\circ} \mathrm{C}$ por 90 minutos para evitar degradação durante o processamento e os corpos de prova foram injetados em uma injetora ROMI 100 TGR, seguindo-se as condições do fabricante do PLA com temperatura de injeção de $200^{\circ} \mathrm{C}$ e temperatura do molde de $25-50{ }^{\circ} \mathrm{C}$.

As composições estão descritas na Tabela 1 de acordo com um experimento fatorial de 2 níveis com ponto central e 2 fatores (MT e MI) e complementada com formulações com aditivos (MT) aplicados isoladamente.

Os ensaios de HDT e Vicat foram realizados segundo as normas ASTM D648-07 e ASTM D1525-09, respectivamente, para um mínimo de três espécimes. Os ensaios de resistência à tração foram realizados segundo norma ASTM D638-10 (corpo de prova tipo I), com velocidade de deformação de $50 \mathrm{~mm} / \mathrm{min}$.

As amostras para o DSC foram retiradas dos corpos de prova injetados. A Temperatura de transição vítrea (Tg), Temperatura de fusão (Tm), Temperatura de cristalização a frio (Tc) foram determinadas utilizando-se um DSC Modelo 2920 TA Instruments, a um taxa de aquecimento
Tabela 1. Composição das amostras segundo o experimento fatorial de dois níveis com ponto central e complementares.

\begin{tabular}{lccc}
\hline \multirow{2}{*}{ Amostra } & \multicolumn{3}{c}{ Concentração(\% m/m) } \\
\cline { 2 - 4 } & PLA & MT & MI \\
\hline PLA0005 & 99,5 & 0 & 0,5 \\
PLA0015 & 98,5 & 0 & 1,5 \\
PLA4005 & 95,5 & 4 & 0,5 \\
PLA4015 & 94,5 & 4 & 1,5 \\
PLA2010 & 97 & 2 & 1 \\
PLA0000 & 100 & 0 & 0 \\
PLA2000 & 98 & 2 & 0 \\
PLA4000 & 96 & 4 & 0 \\
\hline
\end{tabular}

de $10{ }^{\circ} \mathrm{C} / \mathrm{min}$, de $-10{ }^{\circ} \mathrm{C}$ até $220{ }^{\circ} \mathrm{C}$ sob atmosfera de nitrogênio.

Para verificar alterações morfológicas na estrutura cristalina as amostras foram analisadas por Difração de Raios-X utilizando-se um Difratômetro Philips $X^{\prime}$ Pert, varredura de 5 a $50^{\circ}(2 \theta)$, radiação de $\mathrm{K} \alpha$ do $\mathrm{Cu}$ $\left(\lambda=1,54 \mathrm{~A}^{\circ}\right)$, tensão de $40 \mathrm{kV}$ e corrente de $40 \mathrm{~mA}$.

Todas as análises foram realizadas para as amostras antes e após tratamento de recozimento em estufa a $105^{\circ} \mathrm{C}$ por 90 minutos.

\section{Resultados}

Na Tabela 2 são mostrados os valores médios de HDT e Vicat bem como as propriedades térmicas para as amostras antes e após o tratamento de recozimento. A Figura 1 mostra, esquematicamente, como foram definidas as temperaturas obtidas na curva de DSC, como também a entalpia de cristalização a frio $(\Delta \mathrm{Hc})$ e entalpia de fusão $(\Delta \mathrm{Hm})$ que foram utilizadas para o cálculo do grau de cristalinidade.

A Tg medida para o PLA puro (PLA0000) foi de 60 e $65^{\circ} \mathrm{C}$, antes e após o tratamento de recozimento, respectivamente, e a Tm entre 150 e $160{ }^{\circ} \mathrm{C}$, valores dentro das faixas típicas reportadas para o PLA de Tg, entre 50 e $80{ }^{\circ} \mathrm{C}$, e de Tm, entre 130 a $180{ }^{\circ} \mathrm{C}^{[1]}$.

Destaca-se, primeiramente, o comportamento das amostras antes do tratamento térmico. A análise estatística dos resultados para os quais houve a incorporação dos aditivos indicou que os aditivos isolados não causaram alteração significativa nos valores de temperatura HDT e da Vicat. A associação dos dois aditivos indicou uma tendência de redução nestas propriedades térmicas em torno de $5{ }^{\circ} \mathrm{C}$. Os valores de Tg não apresentaram variação significativa em nenhum caso. Nota-se que os valores de HDT e Vicat são encontrados muito próximos da faixa de Tg, como esperado, porém não existe uma correlação entre os valores encontrados. Estes resultados sugerem que as diferenças devem-se às diferentes solicitações que o material sofre para cada método de medição. Para HDT e Vicat, além do aquecimento existe uma solicitação mecânica, enquanto no DSC são detectáveis apenas as alterações no sinal de fluxo de calor. Na literatura são relatados valores de HDT entre $60-66{ }^{\circ} \mathrm{C}$ e Vicat entre 59-60 ${ }^{\circ} \mathrm{C}$ para o PLA semicristalino sendo que este segundo é mais fortemente influenciado pelo grau de cristalinidade do material ${ }^{[9]}$. 
Tabela 2. Valores das propriedades térmicas antes e após recozimento.

\begin{tabular}{|c|c|c|c|c|c|c|c|c|c|c|c|c|}
\hline & \multicolumn{6}{|c|}{ Antes do recozimento } & \multicolumn{6}{|c|}{ Após recozimento } \\
\hline & $\begin{array}{l}\text { HDT } \\
\left({ }^{\circ} \mathbf{C}\right)\end{array}$ & $\begin{array}{l}\text { Vicat } \\
\left({ }^{\circ} \mathbf{C}\right)\end{array}$ & $\begin{array}{c}\mathbf{T g} \\
\left({ }^{\circ} \mathbf{C}\right)\end{array}$ & $\begin{array}{c}\text { Tc } \\
\left({ }^{\circ} \mathbf{C}\right)\end{array}$ & $\begin{array}{c}\text { Tm } \\
\left({ }^{\circ} \mathbf{C}\right)\end{array}$ & $\begin{array}{c}\mathrm{Xc} \\
(\%)\end{array}$ & $\begin{array}{l}\text { HDT } \\
\left({ }^{\circ} \mathbf{C}\right)\end{array}$ & $\begin{array}{l}\text { Vicat } \\
\left({ }^{\circ} \mathbf{C}\right)\end{array}$ & $\begin{array}{c}\mathrm{Tg} \\
\left({ }^{\circ} \mathbf{C}\right)\end{array}$ & $\begin{array}{c}\text { Tc } \\
\left({ }^{(} \mathbf{C}\right)^{2}\end{array}$ & $\begin{array}{c}\mathbf{T m} \\
\left({ }^{\circ} \mathbf{C}\right)\end{array}$ & $\begin{array}{l}\text { Xc } \\
(\%)\end{array}$ \\
\hline PLA0000 & 50 & 60 & 60 & 117 & $151 / 157$ & 3 & 60 & 138 & 65 & --- & $152 / 158$ & 43 \\
\hline PLA0005 & 47 & 59 & 60 & 113 & $153 / 159$ & 0 & 60 & 134 & 57 & --- & $151 / 158$ & 41 \\
\hline PLA0015 & 47 & 58 & 57 & 111 & $151 / 158$ & 0 & 60 & 133 & 63 & --- & $151 / 159$ & 41 \\
\hline PLA2000 & 49 & 58 & 59 & 98 & 157 & 8 & 59 & 129 & 58 & --- & 158 & 44 \\
\hline PLA4000 & 49 & 57 & 60 & 94 & 156 & 12 & 60 & 132 & 60 & --- & 156 & 32 \\
\hline PLA2010 & 45 & 55 & 57 & 96 & 158 & 0 & 60 & 132 & 59 & --- & 157 & 31 \\
\hline PLA4005 & 46 & 56 & 60 & $93 / 112^{1}$ & 156 & 10 & 58 & 132 & 59 & --- & 156 & 31 \\
\hline PLA4015 & 44 & 55 & 56 & $94 / 111^{1}$ & 156 & 9 & 60 & 131 & 59 & --- & 157 & 30 \\
\hline
\end{tabular}

${ }^{1}$ Surgimento de um primeiro pico pronunciado, seguido por um segundo pico pequeno. ${ }^{2}$ Nenhuma amostra apresentou temperatura de cristalização a frio.

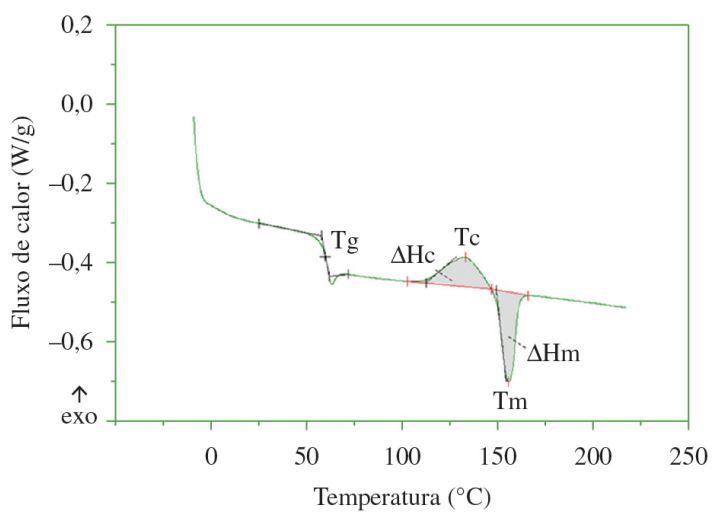

Figura 1. Curva típica do PLA obtida por DSC.

O comportamento da cristalização a frio revela a ação nucleante do MT, pois se observa o deslocamento de Tc para temperaturas mais baixas, indicando o favorecimento do processo de nucleação. O MI não interferiu nesta característica. A associação dos aditivos promove a ocorrência de dois picos de Tc o que sugere a existência de frações do material com diferentes velocidades de cristalização.

Com o objetivo de determinar-se o grau de cristalinidade das amostras injetadas foi necessário descontar a fração que cristalizou durante o aquecimento no DSC. Portanto para o cálculo do grau de cristalinidade (Xc) utilizou-se a Equação 1:

$$
\mathrm{Xc}(\%)=\frac{\Delta \mathrm{Hm}-\Delta \mathrm{Hc}}{\Delta \mathrm{Hm}^{\infty}} \times 100
$$

Onde: $\Delta \mathrm{Hm}$ é a entalpia endotérmica de fusão, $\Delta \mathrm{Hc}$ é a entalpia exotérmica associada aos cristais durante o aquecimento, ou seja, a entalpia da cristalização a frio do PLA e $\Delta \mathrm{H}_{\mathrm{m}}{ }^{\infty}$ é a entalpia teórica do PLA $100 \%$ cristalino que é assumida ser $93 \mathrm{~J} / \mathrm{g}^{[10]}$.

O grau de cristalinidade foi significativamente modificado pelos aditivos. Como esperado o agente nucleante aumentou o grau de cristalinidade de $3 \%$ para o PLA puro para 8 e $12 \%$ com teores de MT de 2 e $4 \%$, respectivamente. $\mathrm{O}$ aditivo modificador de impacto parece restringir a cristalinidade sendo que, pelo método utilizado, o grau de cristalinidade das amostras injetadas mostrou valores zerados. Mesmo quando associado a 2\% do aditivo nucleante o modificador de impacto parece dificultar a cristalização.

Estas diferenças foram também observadas através da transparência das amostras. A Figura 2 mostra as amostras em escala decrescente de cristalinidade, exceto pelo PLA puro (PLA0000) que apresentou em torno de $3 \%$ de cristalinidade e foi o material mais opaco observado. Isto ocorreu, muito provavelmente, por ter poucos cristais porém de maiores dimensões capazes de maior espalhamento da luz.

Os resultados são significativamente alterados após o tratamento de recozimento, sendo este o fator que apresentou maior importância no aumento de todas as propriedades térmicas estudadas. Independentemente da presença de um ou outro aditivo, as amostras recozidas apresentaram valores de HDT entre 58 e $60{ }^{\circ} \mathrm{C}$ e Vicat entre 129 e $138^{\circ} \mathrm{C}$. Isto pode ser atribuído ao aumento da cristalinidade, como é verificado nos valores de Xc. Além disto, pode estar relacionado também com crescimento dos cristais que o processo de recozimento tipicamente confere ao PLA ${ }^{[9]}$. O PLA moldado por injeção pode apresentar-se quase totalmente amorfo devido à lenta cinética de cristalização deste material, o que resulta em peças frágeis e com baixa tenacidade. Estudos anteriores indicam $\mathrm{Tg}$ de $64{ }^{\circ} \mathrm{C}$, Tm de $168{ }^{\circ} \mathrm{C}$ e Xc de $2,7 \%$ para peças injetadas e após recozimento, $\mathrm{Tg}$ de $66{ }^{\circ} \mathrm{C}$, Tm de $169{ }^{\circ} \mathrm{C}$ e Xc de $45 \%$ até $70 \%$ dependendo do peso molecular do polímero ${ }^{[9]}$. Os resultados deste estudo estão em concordância com estes relatos, pois verificou-se pequeno aumento em $\mathrm{Tg}$ e significativo aumento em Xc, além de indicar maior sensibilidade do Vicat ao aumento da cristalinidade do PLA, comparativamente à HDT. Após o recozimento observam-se os seguintes efeitos associados aos aditivos: (a) o MT não demonstra uma tendência clara, indicando que o recozimento é mais determinante quanto ao processo de cristalização do que o efeito nucleante do aditivo; (b) o MI tem uma tendência a reduzir Tg e Xc e (c) a associação dos dois aditivos causa redução em Xc limitando os valores próximos a $30 \%$.

O PLA puro e com a presença do aditivo MI apresentaram pontos de fusão duplos. Isto foi observado em outros estudos sendo fortemente dependentes da taxa de aquecimento ${ }^{[11]}$, sendo explicados pela ocorrência de efeitos competitivos de fusão e recristalização durante o processo de aquecimento. Cristais imperfeitos e pequenos 


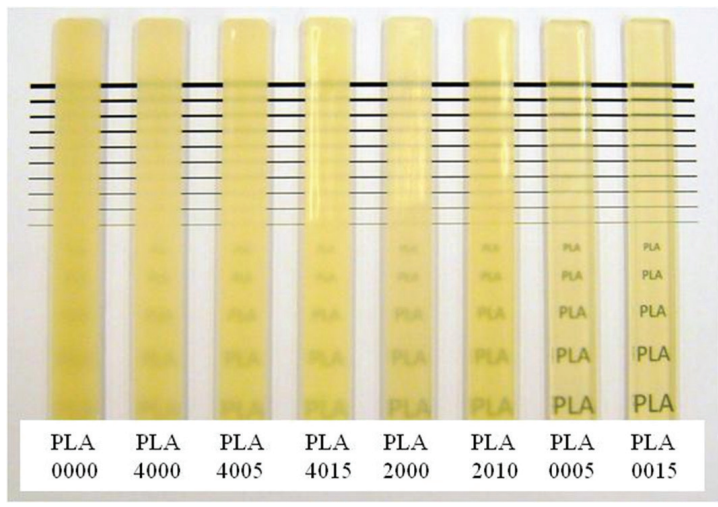

Figura 2. Transparência das amostras de PLA puro e com diferentes teores de MT e MI.

mudam sucessivamente para cristais mais estáveis através deste mecanismo de fusão-recristalização. O pico endotérmico ficaria evidenciado quando a taxa de fusão ultrapassa a de recristalização e um sinal exotérmico quando a taxa de recristalização é maior que a da fusão. Como cada processo tem uma cinética diferente, dependendo das condições de aquecimento um ou outro será mais favorecido ${ }^{[1]}$. Neste trabalho somente a taxa de $10{ }^{\circ} \mathrm{C} / \mathrm{min}$ foi utilizada, e nestas condições este efeito esteve presente. Como isto não ocorreu para todas as amostras pode ser considerado que a presença do aditivo nucleante pode ter alterado a cinética do processo de recristalização ou mesmo proporcionado uma maior homogeneidade no tamanho e perfeição dos cristais originais. Outros autores associaram a presença de dois picos à formação da fase $\beta$ coexistindo com a fase $\alpha^{[12,13]}$, ou mesmo à formação de uma fase desordenada da fase $\alpha$ denominada $\alpha^{\text {'[14] }}$. A análise de difração de raios- $\mathrm{X}$ foi realizada para as amostras antes e após o recozimento, e não foi possível confirmar a coexistência de diferentes fases cristalinas, reforçando a hipótese de que o mecanismo fusão-recristalização estivesse atuando. Similar a outros poliésteres o PLA apresenta polimorfismo com três formas cristalinas principais que dependem das condições de cristalização. A partir do fundido, da cristalização a frio e da solução origina-se a fase $\alpha$, com célula cristalina ortorrômbica com parâmetros $a=1,066 \mathrm{~nm}, b=0,616 \mathrm{~nm}$ e c=2,888nm. Sob diferentes temperaturas de cristalização, uma desordem na fase $\alpha$ também já foi detectada para o PLA, chamada de fase $\alpha$ '. A fase $\beta$ foi detectada na fabricação de fibras, sob condições de alto estiramento e a fase $\gamma$ em condições de cristalização em substrato de hexametilbenzeno ${ }^{[14]}$.

A Figura 3 mostra a diferença entre os gráficos antes e após tratamento de recozimento para o PLA puro. Sem o tratamento térmico, quaisquer formulações resultaram em uma mesma larga banda de absorção. Após o tratamento térmico, para todas as amostras, pode ser observado um pico bem definido em $2 \theta=16,04^{\circ}$ devido à difração dos planos [200] e/ou [110] e picos menores a $2 \theta=14,38^{\circ}$ do plano [010], $2 \theta=18,08^{\circ}$ do plano [203] e $2 \theta=21,86^{\circ}$ do plano [015]. Estes são os picos característicos da fase $\alpha^{[14,15]}$. As amostras modificadas apresentaram o mesmo

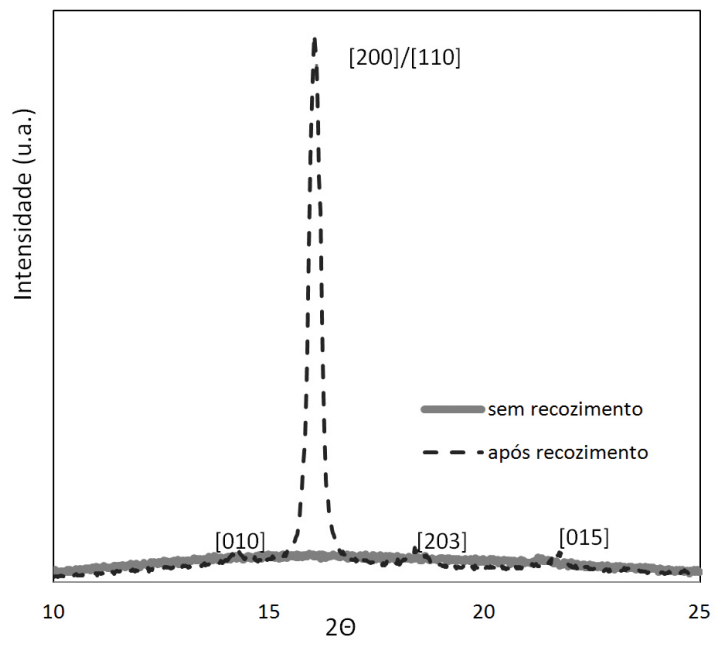

Figura 3. Difratograma do PLA puro antes e após tratamento de recozimento.

Tabela 3. Valores médios do Módulo de elasticidade (E), da Resistência a tração $(\tau)$, e do Alongamento na força máxima $(\varepsilon)$.

\begin{tabular}{ccccccccc}
\hline & \multicolumn{2}{c}{ Antes do recozimento } & & \multicolumn{3}{c}{ Após recozimento } \\
\cline { 2 - 3 } & $\begin{array}{c}\text { E } \\
(\mathbf{M P a})\end{array}$ & $\begin{array}{c}\tau \\
(\mathbf{M P a})\end{array}$ & $\begin{array}{c}\varepsilon \\
(\boldsymbol{\%})\end{array}$ & & $\begin{array}{c}\text { E } \\
(\mathbf{M P a})\end{array}$ & $\begin{array}{c}\tau \\
(\mathbf{M P a})\end{array}$ & $\begin{array}{c}\varepsilon \\
(\boldsymbol{\%})\end{array}$ \\
\hline PLA0000 & 1673 & 60 & 4 & & 1227 & 57 & 3 \\
PLA0005 & 2589 & 65 & 4 & & 2032 & 40 & 2 \\
PLA0015 & 2551 & 64 & 4 & & 2105 & 41 & 2 \\
PLA2000 & 1440 & 54 & 4 & & 1246 & 29 & 2 \\
PLA4000 & 2004 & 53 & 4 & & 1646 & 50 & 3 \\
PLA2010 & 2464 & 55 & 3 & & 1085 & 48 & 3 \\
PLA4005 & 2526 & 58 & 4 & & 1111 & 47 & 3 \\
PLA4015 & 2445 & 55 & 4 & & 902 & 43 & 3 \\
\hline
\end{tabular}

padrão de curva para antes e após recozimento e por isto não estão mostrados os dados para todas as amostras. $\mathrm{A}$ partir destas análises pode-se concluir que a utilização dos aditivos MT e MI não proporcionou alterações na estrutura cristalina do PLA testado onde somente a fase $\alpha$ está presente.

A Tabela 3 apresenta os valores médios do Módulo de elasticidade (E), da Resistência à tração $(\tau)$ e Alongamento na força máxima $(\varepsilon)$ antes e após o tratamento de recozimento.

O Módulo de elasticidade foi afetado significativamente pela adição de MI e MT. O aditivo modificador de impacto, MI, apresentou um comportamento diferente do esperado aumentando em torno de $50 \%$ o valor do Módulo elástico do material. Baixa concentração do agente nucleante reduziu o Módulo elástico que mostrou um aumento quando esta concentração foi aumentada para $4 \%$. Afrifah et al. ${ }^{[16]}$ estudaram o efeito do copolímero etileno/acrilato nas propriedades mecânicas do PLA e observou pouca alteração no módulo elástico, aumento na ductibilidade e redução na resistência à tração somente para concentrações acima de 5\% do aditivo. Neste estudo 
observou-se também uma diminuição no grau de cristalinidade com o aumento no teor do copolímero etileno/acrilato. Estas observações ressaltam o efeito do aditivo em nosso estudo. Os nucleantes, no PLA, podem apresentar efeitos bastante distintos, tendo sido observados aumentos no Módulo elástico de flexão para PLA nucleado com talco e uma manutenção dos valores quando o nucleante foi o elileno bis-esteramida $(\mathrm{EBS})^{[10]}$. A associação dos dois aditivos teve um efeito muito positivo com aumento de $50 \%$ no valor de E. A adição de MT e MI mostra pequena influência sobre a Resistência à tração e sobre o Alongamento dos corpos de prova testados. O fator recozimento, diferentemente do esperado, causou redução em todas as propriedades. A maior cristalinidade observada deveria ter aumentado o Módulo elástico, bem como a Resistência à tração ${ }^{[10]}$, o que não ocorreu. Isto poderia ser explicado por uma possível degradação causada pelas condições empregadas no tratamento térmico.

\section{Conclusões}

O uso dos aditivos propostos não se mostrou viável para o aumento da HDT da Vicat do PLA estudado. Estas propriedades mostraram-se sensíveis ao aumento do grau de cristalinidade. Este foi significativamente modificado pelos aditivos, porém o aumento de 3\% do PLA puro para algo em torno de $12 \%$ do PLA modificado não foi suficiente para promover as melhorias esperadas. $\mathrm{O}$ recozimento foi mais efetivo promovendo aumento no grau de cristalinidade para valores de até $44 \%$ e elevou os valores de HDT em torno de $10{ }^{\circ} \mathrm{C}$ e de Vicat em mais de $70{ }^{\circ} \mathrm{C}$.

As modificações no estado cristalino também foram acompanhadas pela transparência das amostras e por Difração de Raios-X que identificou a predominância da fase $\alpha$, caracterizada por uma célula unitária ortorrômbica.

A Tg foi pouco alterada, sendo observados uma tendência de redução pela presença dos aditivos e um discreto aumento pelo recozimento. A cristalização a frio do PLA foi acelerada pelo aditivo nucleante e totalmente eliminada pelo tratamento de recozimento.

O Módulo de elasticidade apresentou aumento significativo pela adição dos aditivos testados, o que não foi observado para a Resistência à tração e para o Alongamento. O recozimento também foi significativo, porém, diferentemente do esperado, causou redução em todas as propriedades mecânicas. A maior cristalinidade observada deveria ter aumentado o Módulo elástico, bem como a Resistência à tração, o que não ocorreu por uma possível degradação causada pelas condições empregadas no tratamento térmico.

Para potenciais aplicações industriais os teores adequados de aditivos e uma análise detalhada do processo ainda são necessários, incluindo-se o tempo e temperatura de resfriamento durante o processo de moldagem, para que o material final possa apresentar as propriedades desejadas.

\section{Referências Bibliográficas}

1. Auras, R.; Harte, B. \& Selke, S. - Macromol. Biosci., 4, p.835 (2004). PMid:15468294. http://dx.doi.org/10.1002/ mabi.200400043

2. Nampoothiri, K. M.; Nair, N. R. \& John, R. P. - Bioresour. Technol. 101, p.8493 (2010).

3. Suryanegara, L.; Nakagaito, A. N. \& Yano, H. - Compos. Sci. Technol., 69, p.1187 (2009). http://dx.doi. org/10.1016/j.compscitech.2009.02.022

4. Hughes, J.; Thomas, R.; Byun, Y. \& Whiteside, S. - Carbohyd. Polym., 88, p.165 (2012). http://dx.doi. org/10.1016/j.carbpol.2011.11.078

5. Hassouna, F.; Raquez, J-M.; Addiego, F.; Toniazzo, V.; Dubois, P. \& Ruch, D. - Eur. Polym. J., 48, p.404 (2012). http://dx.doi.org/10.1016/j.eurpolymj.2011.12.001

6. Kumar, M.; Mohanty S.; Nayak, S. K. \& Parvaiz, M. R. - Bioresour. Technol., 101, p.8406 (2010). PMid:20573502. http://dx.doi.org/10.1016/j.biortech.2010.05.075

7. Yang, S-1.; Wu, Z-H.; Yang, W. \& Yang, M-B. - Polym. Test., 27, p.957 (2008). http://dx.doi.org/10.1016/j. polymertesting.2008.08.009

8. Pantani, R.; De Santis, F.; Sorrentino, A.; De Maio, F. \& Titomanlio, G. - Polym. Degrad. Stab., 95, p.1148 (2010). http://dx.doi.org/10.1016/j.polymdegradstab.2010.04.018

9. Perego, G. \& Cella, G. D. - "Mechanical Properties", in: Poly(lactic acid): Synthesis, Structures, Properties, Processing, and Applications", John Wiley \& Sons (2010). http://dx.doi.org/10.1002/9780470649848.ch11

10. Harris, A. M. \& Lee, E. C. - J. Appl. Polym. Sci., 107, p.2246 (2008). http://dx.doi.org/10.1002/app.27261

11. Yasuniwa, M.; Tsubakihara, S.; Sugimoto, Y. \& Nakafuku, C. - J. Polym. Sci., Part B: Polym. Phys., 42, p.25 (2004). http://dx.doi.org/10.1002/polb.10674

12. Fambri, L. \& Migliaresi, C. - "Crystallization and Thermal Properties", in: Poly(lactic acid): Synthesis, Structures, Properties, Processing, and Applications, John Wiley \& Sons (2010). http://dx.doi.org/10.1002/9780470649848. ch9

13. Hoogsteen, W.; Postema, A. R.; Pennings, A. J. \& Brinke, G. - Macromolecules, 23, p.634 (1990). http://dx.doi. org/10.1021/ma00204a041

14. Di Lorenzo, M. L.; Cocca, M. \& Malinconico, M. - Thermochim. Acta, 522, p.110 (2011). http://dx.doi. org/10.1016/j.tca.2010.12.027

15. Mano, J. F.; Wang, Y.; Viana, J. C.; Denchev, Z. \& Oliveira, M. J. - Macromol. Mater. Eng., 289, p.910 (2004). http:// dx.doi.org/10.1002/mame.200400097

16. Afrifah, K. A. \& Matuana, L. M. - Macromol. Mater. Eng., 295, p.802 (2010). http://dx.doi.org/10.1002/ mame. 201000107

Enviado: $28 / 09 / 12$

Reenviado: $24 / 06 / 13$

Aceito: $27 / 06 / 13$ 